\title{
Vertical dispersion of light inertial particles in stably stratified turbulence : the influence of the Basset force
}

Citation for published version (APA):

Aartrijk, van, M., \& Clercx, H. J. H. (2010). Vertical dispersion of light inertial particles in stably stratified turbulence : the influence of the Basset force. Physics of Fluids, 22(1), 013301-1/9. [013301].

https://doi.org/10.1063/1.3291678

DOI:

10.1063/1.3291678

Document status and date:

Published: 01/01/2010

Document Version:

Publisher's PDF, also known as Version of Record (includes final page, issue and volume numbers)

Please check the document version of this publication:

- A submitted manuscript is the version of the article upon submission and before peer-review. There can be important differences between the submitted version and the official published version of record. People interested in the research are advised to contact the author for the final version of the publication, or visit the $\mathrm{DOI}$ to the publisher's website.

- The final author version and the galley proof are versions of the publication after peer review.

- The final published version features the final layout of the paper including the volume, issue and page numbers.

Link to publication

\section{General rights}

Copyright and moral rights for the publications made accessible in the public portal are retained by the authors and/or other copyright owners and it is a condition of accessing publications that users recognise and abide by the legal requirements associated with these rights.

- Users may download and print one copy of any publication from the public portal for the purpose of private study or research.

- You may not further distribute the material or use it for any profit-making activity or commercial gain

- You may freely distribute the URL identifying the publication in the public portal.

If the publication is distributed under the terms of Article 25fa of the Dutch Copyright Act, indicated by the "Taverne" license above, please follow below link for the End User Agreement:

www.tue.nl/taverne

Take down policy

If you believe that this document breaches copyright please contact us at:

openaccess@tue.nl

providing details and we will investigate your claim. 


\title{
Vertical dispersion of light inertial particles in stably stratified turbulence: The influence of the Basset force
}

\author{
M. van Aartrijk and H. J. H. Clercx ${ }^{a)}$ \\ Department of Physics, Fluid Dynamics Laboratory, International Collaboration for Turbulence Research \\ (ICTR) and J. M. Burgers Center for Fluid Dynamics, Eindhoven University of Technology, \\ P.O. Box 513, 5600 MB Eindhoven, The Netherlands
}

(Received 17 March 2009; accepted 10 December 2009; published online 15 January 2010)

\begin{abstract}
The dispersion of light inertial particles $\left[\rho_{p} / \rho_{f}=\mathcal{O}(1)\right]$ in statistically stationary stably stratified turbulence is studied by means of direct numerical simulations. The light particle dispersion behavior is found to be comparable to that of heavy particles when displayed as a function of the Stokes number. Deviations from fluid particle dispersion are found already for small Stokes numbers; the length of the typical plateau for vertical dispersion is shorter for the light inertial particles. All the forces in the Maxey-Riley equation are taken into account and they are found to be of similar magnitude as the Stokes drag for particles with $\rho_{p} / \rho_{f}=\mathcal{O}(1)$. However, not all forces directly influence the particle dispersion. It is shown that especially the often neglected Basset force plays a considerable role in the vertical dispersion of light particles in stratified turbulence. Neglecting this force results in an overprediction of the vertical dispersion by about $15 \%-20 \%$.

(C) 2010 American Institute of Physics. [doi:10.1063/1.3291678]
\end{abstract}

\section{INTRODUCTION}

The vertical and horizontal dispersion of inertial particles plays an important role in geophysical flows. The behavior of small particles, such as plankton, algae, and larger aggregates of marine particles in coastal areas, estuaries, and lakes is affected by the properties of the flow. The turbulent flow field determines the spatial distribution of plankton and algae, and with that their possibility to encounter nutrients or predators. It also determines the absorption of sunlight necessary for photosynthesis at different heights within the fluid column. ${ }^{1}$ Moreover, the various hydrodynamic forces act at the smallest scales of turbulent flows at which they can influence particle interaction, collisions and aggregate formation.

In natural turbulent flows often a stable density stratification is present. In these stably stratified flows the vertical density gradient is negative; the average density of the fluid is decreasing with height. Strongly stratified flows typically display thin layers of large quasihorizontal vortical structures with strong shearing between these layers. Moreover, internal gravity waves are present in stably stratified flows. See, for example, the works by Riley and LeLong ${ }^{2}$ and Brethouwer $e t a l .^{3}$ for detailed descriptions of stably stratified turbulence.

Dispersion studies in stably stratified turbulence mainly focused on fluid particles that exactly follow the flow, see for example, Refs. 4-7. In a previous paper we presented the dispersion of heavy inertial particles in these stratified turbulent flows. ${ }^{8}$ The negative vertical density gradient suppresses vertical fluid motions. As a result, also the vertical dispersion of (inertial) particles is reduced compared with that in isotropic turbulence. Fluid particles that are displaced from their

\footnotetext{
a)Electronic mail: h.j.h.clercx@tue.nl.
}

original equilibrium height show a strong tendency to return to that equilibrium height due to a restoring buoyancy force. The vertical mean-squared displacement of fluid particles displays three successive regimes: for short times the classical $t^{2}$-regime (ballistic regime) ${ }^{9}$ is obtained and for intermediate times a plateau can be identified which scales with the buoyancy frequency as $N^{-2}{ }^{6}$ For long times, the dispersion behavior depends on whether decaying or forced stratified turbulence is studied. In forced, and therefore statistically stationary, stably stratified turbulence a diffusion limit is obtained where the dispersion is proportional to $\mathcal{O}(t)$. This diffusive regime is caused by molecular diffusion of the active scalar (density) and it is only observed for statistically stationary stably stratified turbulence. ${ }^{4,10}$ Recently, Lindborg and Brethouwer ${ }^{11}$ reported a theoretical derivation of the scaling behavior in the intermediate $N^{-2}$-regime and of the irreversible mixing in the diffusive regime. Also in the horizontal direction the dispersion of fluid particles in stably stratified turbulence is altered. Compared with that in isotropic turbulence it is similar for short times, but for long times it is enhanced. In the long-time limit it is found to scale proportional to $t^{2.1 \pm 0.1}$, larger than the classical linear diffusion limit. ${ }^{4}$ This enhanced horizontal dispersion results from the strong local vertical shear that is present in strongly stratified turbulence.

When inertial particles are considered, several forces act on the individual particles. These forces are described in the Maxey-Riley equation. ${ }^{12}$ In the limit of small heavy particles, with densities $\rho_{p}$ much larger than that of the surrounding fluid $\left(\rho_{f}\right)$ (for example, aerosols in air), only drag forces and gravity are relevant. The other forces can be neglected. For these heavy particles mainly the vertical dispersion is altered by the inertia. Increasing the particle's inertia, expressed using the Stokes number St, results in a less pronounced plateau which even vanishes for Stokes numbers of 
$\mathcal{O}(10)$ and higher. In the long-time limit, the vertical dispersion increases with increasing St and the scaling behavior becomes proportional to $t$. The increased vertical dispersion with increasing Stokes number is a result of the inertial forces that start to dominate the restoring buoyancy force of the fluid. In the horizontal direction the long-time dispersion seems hardly affected by varying the Stokes number, and its scaling behavior is similar to that of fluid particles in stratified turbulence. ${ }^{8}$

An additional effect of the inertia of the particles is that they are not uniformly distributed over the flow domain. This so-called effect of preferential concentration, which is maximum for Stokes numbers around one ${ }^{13}$ leads to biased particle trajectories. Particles tend to be present preferentially in flow regions with low vorticity.

Not much work is available in the literature about the dispersion of light inertial particles in turbulent flows. Single plankton or algal particles have a very small Stokes number and their dispersion behavior in stratified turbulence might therefore be well represented by fluid particle dispersion. These small microorganisms, however, can form aggregates with Stokes numbers at which the forces that are acting on the particles cannot be neglected. For the light particles $\left[\rho_{p} / \rho_{f}=\mathcal{O}(1)\right]$ all forces in the Maxey-Riley equation need to be taken into account. The topic is studied by Armenio and Fiorotto $^{14}$ for turbulent channel flows. They conclude that apart from drag forces also the pressure gradient and the Basset force are important terms in the particle equation of motion, when looking at the magnitude of the forces. The effect on the particle dispersion of the forces other than the Stokes drag force, however, is found to be very small. ${ }^{14}$ Other studies that might be relevant for the stratified turbulence that is considered in this paper, are the works by Tanga and Provenzale ${ }^{15}$ and by Candelier et al. ${ }^{16}$ They both studied light particle behavior in vortical structures. A common observation in these works is that the Basset force tends to trap the particles for longer times within a vortex.

In this contribution we report on the dispersion of light inertial particles in statistically stationary stably stratified turbulence. It will be discussed whether the dispersion behavior of light particles is the same as for heavy particles (by using data for heavy particle dispersion which has been published previously ${ }^{8}$ ). Furthermore, the influence of the individual forces in the Maxey-Riley equation on the particle dispersion will be examined.

The paper is organized as follows. First the numerical method used in this investigation is introduced in Sec. II. Hereby, it is mainly focused on the Lagrangian part of the code, which computes the particle trajectories through the flow. Next, in Sec. III the dispersion results are discussed. Section III A compares dispersion statistics of light and heavy particles. The influence of the different forces on light particle dispersion in stratified turbulence is subsequently discussed in Sec. III B. Whether these results are also manifested in isotropic turbulence or for heavy particles, will be examined in Sec. III C. This section furthermore discusses the influence of a mean settling velocity resulting from gravity on the main conclusions. Concluding remarks are given in Sec. IV.

\section{NUMERICAL METHOD}

This study is performed by means of direct numerical simulations. The numerical code consists of two parts. The flow field is solved using the Eulerian approach and the Lagrangian approach is applied to study the particle trajectories. First, the Eulerian part will be discussed briefly. Next, the Lagrangian part will be described in more detail.

\section{A. DNS of the Boussinesq equations}

The Navier-Stokes equations within the Boussinesq approximation are solved on a triple-periodic domain using a pseudospectral code. ${ }^{4,17}$ A linear stable background density stratification is imposed, which is kept constant throughout a simulation. Density fluctuations are present on top of the linear profile, and the total density is given by $\rho_{f}=\rho_{0}+\bar{\rho}(z)$ $+\rho^{\prime}(x, y, z, t)\left(\rho_{0}\right.$ a reference value, $\bar{\rho}$ the background profile, $\rho^{\prime}$ the density fluctuations). From the density gradient the buoyancy frequency $N^{2}=-\left(g / \rho_{0}\right)(\partial \bar{\rho} / \partial z)$ can be computed, with $g$ the gravitational acceleration. The resulting flow field is homogeneous and anisotropic. In order to keep the stratified turbulence statistically stationary, large-scale forcing is applied. A detailed description of the Eulerian part of the code, the forcing protocol and the resulting flow fields can be found in Refs. 4, 8, and 13.

The flow field under consideration, with $N=0.31 \mathrm{~s}^{-1}$, will be called case N10 in correspondence with Ref. 8. This stratification strength is realistic for stratified marine environments. ${ }^{18}$ An impression of the degree of anisotropy of the flow is given by the ratios $L_{z} / L_{h}=0.16, u_{h} / u_{\mathrm{rms}}=1.15$, and $w_{\mathrm{rms}} / u_{\mathrm{rms}}=0.40$. Herein, $L_{z}$ and $L_{h}$ are the vertical and horizontal integral length scales, and $u_{h}, w_{\text {rms }}$, and $u_{\text {rms }}$ are the horizontal, vertical and overall rms-velocity, respectively. According to the classification proposed by Brethouwer et $a .^{3}$ our flow can be called weakly stratified turbulence. In this regime, where the modified Reynolds number $R=\operatorname{Fr}_{h}^{2} \operatorname{Re}>1$ (Reynolds number $\operatorname{Re}$ is 388 and horizontal Froude number $\mathrm{Fr}_{h}=0.11$, giving $R=4.7$ ), large-scale quasihorizontal layers are observed but at the same time smallscale turbulentlike motion and overturning are present in the flow. ${ }^{19}$

\section{B. Particle tracking}

When a statistically stationary flow field is obtained, particles are released at random positions in the domain. Three types of particles are tracked in the flow: fluid particles $\left(\rho_{p}=\rho_{f}\right.$ and St $\left.\rightarrow 0\right)$, light particles $\left(\rho_{p} / \rho_{f}=\rho^{*}=\mathcal{O}(1)\right.$, but St not necessarily zero) and heavy particles $\left(\rho_{p} \gg \rho_{f}\right)$, with $\rho_{p}$ the particle density. The fluid particle and heavy inertial particle results will be used as a reference. Particle trajectories are calculated according to

$$
\frac{d \boldsymbol{x}_{p}(t)}{d t}=\boldsymbol{u}_{p}(t)
$$

with $\boldsymbol{x}_{p}$ the particle position and $\boldsymbol{u}_{p}$ its velocity. For fluid particles their velocities are derived from cubic spline interpolation of the velocity field at the particle position. ${ }^{4}$ The 
velocities of the inertial particles (light and heavy) are obtained by solving the Maxey-Riley equation ${ }^{12}$

$$
\begin{aligned}
m_{p} \frac{d \boldsymbol{u}_{p}}{d t}= & 6 \pi a \mu\left(\boldsymbol{u}-\boldsymbol{u}_{p}+\frac{1}{6} a^{2} \nabla^{2} \boldsymbol{u}\right)+m_{f} \frac{D \boldsymbol{u}}{D t}+\left(m_{p}-m_{f}\right) \\
& \times \boldsymbol{g}+\frac{1}{2} m_{f}\left(\frac{D \boldsymbol{u}}{D t}-\frac{d \boldsymbol{u}_{p}}{d t}+\frac{1}{10} a^{2} \frac{d}{d t} \nabla^{2} \boldsymbol{u}\right) \\
& +6 \pi a^{2} \mu \int_{0}^{t} d \tau \frac{d \boldsymbol{u} / d \tau-d \boldsymbol{u}_{p} / d \tau+\frac{1}{6} a^{2} d \nabla^{2} \boldsymbol{u} / d \tau}{[\pi \nu(t-\tau)]^{1 / 2}}
\end{aligned}
$$

The particle mass is given by $m_{p}, a$ is the radius of the particle, and $m_{f}$ is the mass of a fluid element with a volume equal to that of the particle. The fluid velocity at the particle position is denoted by $\boldsymbol{u}, \nu$ is the kinematic viscosity, and $\mu=\nu \rho_{f}$ is the dynamic viscosity. The forces on the right-hand side of this equation are viscous drag, a local pressure gradient in the undisturbed fluid, gravitational forces, added mass and the Basset history force, successively. The terms including $\nabla^{2} \boldsymbol{u}$ are the Faxén corrections to the respective forces, which take into account the nonuniformity of the flow field at scales comparable to the particle's diameter. For the added mass term the form described by Auton $e t a l .^{20}$ is used (see Sec. III C for a discussion concerning modified longtime memory kernels).

The Maxey-Riley equation is derived for small $\left(d_{p} \ll \eta\right.$ with $d_{p}=2 a$ the particle diameter and $\eta$ the Kolmogorov length) isolated rigid spheres, under the assumption of a low particle Reynolds number ${ }^{12} \operatorname{Re}_{p}=\left(\left|\boldsymbol{u}-\boldsymbol{u}_{p}\right| d_{p}\right) / \nu \ll 1$. In the limit of heavy particles Eq. (2) reduces to

$$
\frac{d \boldsymbol{u}_{p}}{d t}=\frac{1}{\tau_{p}}\left(\boldsymbol{u}-\boldsymbol{u}_{p}\right)+\boldsymbol{g}
$$

A measure of the particle inertia is the particle response time $\tau_{p}=\left[d_{p}^{2}\left(\rho_{p} / \rho_{f}\right) / 18 \nu\right]$. The particle inertia will be expressed in the following using the Stokes number $\mathrm{St}=\tau_{p} / \tau_{K}$, with $\tau_{K}$ the Kolmogorov time. For most of the results presented in this paper the body force on the particles due to gravity is set to zero $(g=0)$. In Sec. III C we will consider the influence of a mean settling velocity of the light particles resulting from gravity.

Since we are using small particles $\left(d_{p} \leq \eta\right)$ and low particle volume fractions, the influence of the particles on the flow field and particle-particle interactions are assumed negligible and the system is called one-way coupled. The initial velocities of the particles are set equal to the local fluid velocity. Inertial particles do not exactly follow the flow and it takes about two eddy turnover times $T_{E}$ before the particles are completely adapted and reach a quasisteady distribution. From this time on the strength of the effect of preferential concentration remains constant. The calculation of statistical quantities starts only after this initial transient. Statistics are computed from 4000 trajectories in case of light particles, since these computations are computationally expensive. One run with 20000 particles is performed in order to check the accuracy of the statistics. For all heavy particle simula- tions 20000 trajectories are obtained. Velocity and position time series of the particles are collected for about ten eddy turnover times.

Time integration of Eq. (1) is performed using the thirdorder Adams-Bashforth method. Due to high memory requirements when solving the equation of motion for the particles, it is chosen to use the second-order Adams-Bashforth method for the numerical integration of Eq. (2) or (3). In order to solve Eq. (2) [or (3)] the fluid velocity and its firstand second-order spatial derivatives at the particle position are needed. Cubic spline interpolation is implemented in the code for this purpose. Furthermore, time derivatives of both the fluid velocity and the particle velocity are present in Eq. (2). Central differences are used to obtain the time derivatives, only for $d \boldsymbol{u}_{p} / d t$ at time $t-\Delta t$ ( $\Delta t$ the time step) the method of backward differences is chosen. The various discretization methods are elaborately tested. The integral in the Basset force is converted into a sum over a finite number of time steps. It has been tested how many previous data points are needed for the Basset force to reproduce this force accurately. Since the particles are small, the smallest scales of the flow are the most important for the strength of the forces that act on a particle. It is found that the history term has to be calculated over a time interval of at least one Kolmogorov time. For the runs presented here a history of about $2 \tau_{K}$ is chosen (corresponding to 500 time steps); increasing this time does not significantly change any of the forces acting on a particle and thus also not the dispersion results. The time step taken for the integration of the particle position and velocity is the same as for the Eulerian velocity field, the Courant-Friedrichs-Lewy condition is the most stringent for the choice of the time step. It has been tested that the strength of the different forces on the particles or statistical properties such as particle dispersion does not depend on the chosen time step.

When studying light particle dispersion in stably stratified turbulence, the density gradient in the flow must be taken into account. Because the background density of the fluid is not constant in the vertical direction and the particle density is close to the fluid density, care needs to be taken in calculating particle statistics. The density ratio $\rho^{*}$ is changing continuously. Moreover, the background density profile $\bar{\rho}$ is not periodic in the vertical direction.

The approach that is therefore adopted is the following. The particles are released in the portion of the domain between $0.25 L_{0, z}$ and $0.75 L_{0, z}$ with $L_{0, z}$ the domain size in vertical direction. It is seen previously for fluid particles and for low Stokes number heavy particles ${ }^{4,8}$ that the vertical radius of action of the particles is limited when no gravitational forces are acting on the particles themselves. By keeping them away from the top and bottom boundaries initially, they will not cross these boundaries and it can be avoided that they enter a region with a completely different background density during a simulation. All particles are assigned a certain density ratio at their release in the flow. The initial density ratios $\rho^{*}$ of the six different light particle ensembles that 
TABLE I. Properties of the particles that are studied in stably stratified turbulence. The Stokes numbers given here are based on the initial values for $\rho^{*} . M$ is the number of particles that are tracked during a time $T_{\text {tr }}$ after reaching a steady state distribution. $\mathrm{L}=$ light and $\mathrm{H}=$ heavy. Heavy particle data is reported previously in Ref. 8.

\begin{tabular}{lccccc}
\hline \hline Particle & $\rho^{*}$ & $d_{p}$ & $\mathrm{St}$ & $M$ & $T_{\mathrm{tr}}$ \\
\hline L1 & 1.01 & $\eta$ & 0.06 & 4000 & $10 T_{E}$ \\
L2 & 1.4 & $\eta$ & 0.08 & 4000 & $10 T_{E}$ \\
L3 & 5.0 & $\eta$ & 0.28 & 4000 & $10 T_{E}$ \\
L4 & 10.0 & $\eta$ & 0.55 & 4000 & $10 T_{E}$ \\
L5 & 25.0 & $\eta$ & 1.38 & 4000 & $10 T_{E}$ \\
L6 & 144.4 & $0.26 \eta$ & 0.55 & 4000 & $10 T_{E}$ \\
H1 & 13500 & $0.020 \eta$ & 0.30 & 20000 & $40 T_{E}$ \\
H2 & 13500 & $0.033 \eta$ & 0.80 & 20000 & $40 T_{E}$ \\
H3 & 13500 & $0.049 \eta$ & 1.81 & 20000 & $40 T_{E}$ \\
H4 & 13500 & $0.095 \eta$ & 6.75 & 20000 & $40 T_{E}$ \\
\hline \hline
\end{tabular}

are tracked, are given in Table I. Depending on their vertical position in the flow field and thus on the density of the surrounding fluid, the corresponding particle density is calculated from $\rho_{p}=\rho_{f} \rho^{*}$. The density of the fluid at the particle position is computed from $\rho_{f}\left(\boldsymbol{x}_{p}\right)=\rho_{0}+\bar{\rho}\left(z_{p}\right)+\rho^{\prime}\left(\boldsymbol{x}_{p}\right)$. The value for the fluctuating component of the density is obtained using the same cubic spline interpolation technique as is used for the fluid velocity. In the remainder of a simulation the density of the particles is kept fixed. The diameter of the particles is also kept fixed throughout a simulation. Due to the vertical motion of the particles their density ratio is changing in time. This change, however, is small. The total density gradient over the computational domain is about $1 \%$ of $\rho_{0}$. Suppose that the maximum vertical displacement of a particle is one-tenth of the domain, then the change in the background density will be only about 1 per mille, apart from any density fluctuations $\rho^{\prime}$. Because of the small changes in the density ratio also the Stokes number of the particles fluctuates in time, again these fluctuations are of $\mathcal{O}\left(10^{-3}\right)$.

For the heavy particles the changes in the background density of the fluid can be neglected compared with the density ratio $\rho_{p} / \rho_{f}$, which is of order $10^{4}$. These heavy particles are released throughout the whole domain and both their density and density ratio remain constant throughout a simulation.

The properties of the particles are given in Table I. The range of particle parameters that can be investigated for the light particles is limited. To stay close to the applications in mind, density ratios of about 1 (plankton, algae) or 2.7 (sand) are preferred. The particle diameter is restricted to $\mathcal{O}(\eta)$ for the Maxey-Riley equation to remain valid. The Stokes number $\mathrm{St}=\tau_{p} / \tau_{K}$ of a particle is a function of $d_{p}, \rho^{*}, \nu$, and $\tau_{K}$. The latter two parameters are determined by the computation of the flow field. For $d_{p} \lesssim \eta$ and small density ratios, the resulting Stokes numbers are also small (smaller than about 1).

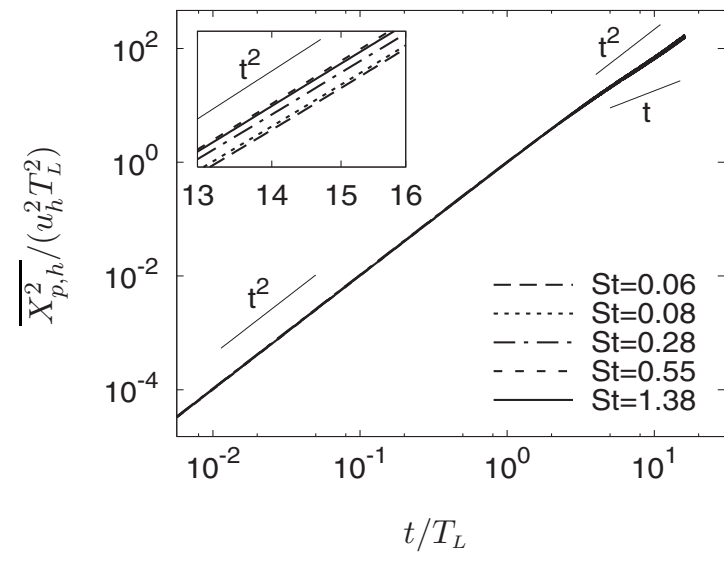

FIG. 1. Mean-squared displacement of light particles in horizontal direction for stably stratified turbulence with $N=0.31 \mathrm{~s}^{-1}$. The results are plotted for the particles denoted by L1-L5 in Table I (no gravity acting on the particles). The inset shows the long-time behavior; for this range of Stokes numbers the horizontal dispersion increases with increasing Stokes number. For the scaling of the horizontal dispersion the Lagrangian time scale $T_{L}$ is used.

\section{RESULTS}

What parameter determines the dispersion of inertial particles in stably stratified turbulence? Which forces play a role in this dispersion process? Also, are these results unique for stably stratified turbulence and for light particles? These topics will be discussed in the following sections.

\section{A. Light particle dispersion versus heavy particle dispersion}

The dispersion of light inertial particles in stably stratified turbulence is shown in Fig. 1 for the horizontal direction and in Fig. 2 for the vertical direction. The dispersion is here defined as the mean-squared displacement of the particles in $i$-direction $(i \in\{x, y, z\})$, computed from the position time series of the particles according to

$$
\overline{X_{p, i}^{2}}(t)=\frac{1}{M} \sum_{q=1}^{M}\left[x_{p, i}(t)-x_{p, i}(0)\right]_{q}^{2},
$$

with $M$ the number of particles. The two horizontal components of the dispersion ( $x$ and $y$ ) are similar and only an averaged result will be presented here for the horizontal direction, denoted with the subscript $h$. The horizontal dispersion is thus expressed as $\overline{X_{p, h}^{2}}=\frac{1}{2}\left(\overline{X_{p, x}^{2}}+\overline{X_{p, y}^{2}}\right)$.

For both the horizontal and the vertical dispersion the results are similar to those obtained for heavy particles in stratified turbulence. ${ }^{8}$ In the horizontal direction the dispersion scales as $t^{2}$ for short times and for longer times the dispersion is clearly enhanced compared with the dispersion in isotropic turbulence. For both types of particles (heavy + light) the long-term horizontal dispersion scales proportional to $t^{\alpha}$ with $\alpha \approx 2$. As can be seen in the inset of Fig. 1 the long-time dispersion increases with increasing Stokes number for the range of Stokes numbers studied here $(\mathrm{St} \lesssim 1)$. 

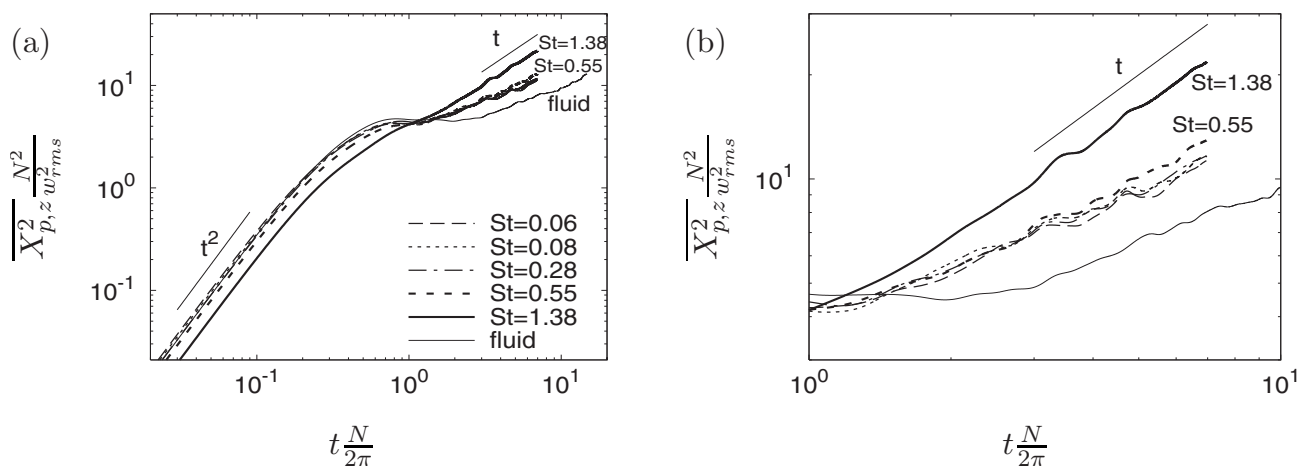

FIG. 2. Vertical mean-squared displacement of light particles for stably stratified turbulence with $N=0.31 \mathrm{~s}^{-1}$. The results are plotted for the particles denoted by L1-L5 in Table I (no gravity acting on the particles). For reference the results for fluid particles are added. The long-time regime is emphasized in (b).

Also in the vertical direction the same trend is visible as for heavy particles. The graphs of the smallest three Stokes numbers shown in Fig. 2 more or less collapse and their dispersion behavior is similar to that of fluid particles. Only the length of the plateau is shorter for the light particles. This can be an indication that also at these small Stokes numbers the different forces affect light particle dispersion. It would be in contradiction with the assumption that is often used in the literature that the dispersion of inertial particles with $\mathrm{St} \ll 1$ can be described using fluid particles. For Stokes numbers larger than about 0.5 the graphs start to deviate. A reduced rms-velocity results in a reduced dispersion initially. For longer times it can be seen that the plateau becomes less pronounced and that the dispersion increases with increasing St.

The comparison between the vertical dispersion for light and heavy particles is shown in Fig. 3. For the heavy particles the simplified Eq. (3) is solved. For both the heavy particles and the light particles the tendency to show a plateau decreases with increasing St. Besides, the results for the light particles, with $\mathrm{St}=0.55$ and $\mathrm{St}=1.38$, nicely match the heavy particle results in the long-time limit, which shows an increasing dispersion with increasing St. The particle's inertia becomes more important than the restoring buoyancy force of the fluid. Comparing light and heavy particles with similar Stokes number $[\mathrm{St} \approx 0.30$, Fig. 3(b)] shows the same observation. The small differences can be caused, among others, by the different initial particle distribution over the computational domain and the different number of particles used to compute statistics.

The Stokes number is thus a good parameter to express the influence of inertia on the particle dispersion; using this parameter the results of particles with widely differing properties such as those of the light and heavy particles show a similar trend. However, the Stokes number not fully determines the dispersion of (light) inertial particles in stably stratified turbulence, also the separate particle parameters $\rho^{*}$ and $d_{p}$ play a small role. In order to investigate the influence of these parameters also particles L6, with the same Stokes number as particle ensemble L4 but with different $\rho^{*}$ and $d_{p}$, are tracked. The results of particles L4 and L6 differ. In both the horizontal and the vertical direction the dispersion of the smallest and heaviest of the two types of particles (L6) is larger. The differences are of the order of 5\%-10\% and they are a clear indication that it is not the Stokes number alone that determines all the dispersion details. ${ }^{19}$

\section{B. Influence of the individual forces on light particle dispersion}

The dispersion results for light particles with small Stokes numbers differ from those obtained for fluid particles, as seen in Fig. 2. Furthermore, it is pointed out in Sec. III A that the individual parameters $\rho^{*}$ and $d_{p}$ affect the dispersion
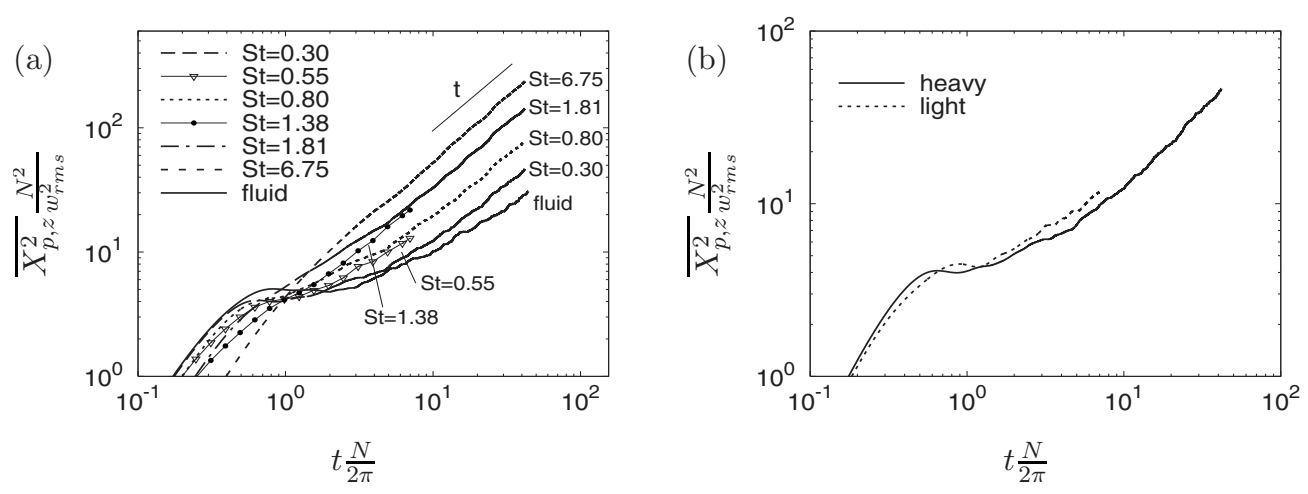

FIG. 3. Vertical single-particle dispersion for fluid particles, light particles (particles L4 and L5 in Table I, with $\mathrm{St}=0.55$ and $\mathrm{St}=1.38$ ) and heavy particles. In (a) the lowest curve (long-time limit) shows the fluid particle result. No gravity is acting on the particles. The initial $t^{2}$-range is left out in order to focus on the plateau and the long-time regime. (b) compares the results for light (L3) and heavy (H1) particles with similar Stokes number (St $\approx 0.30$ ). 


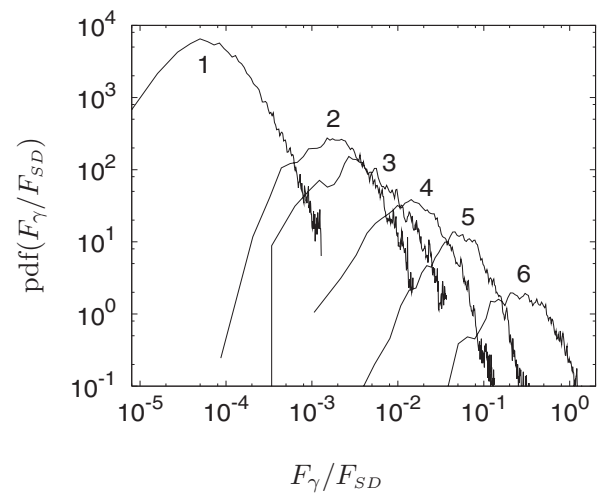

FIG. 4. PDF of the ratio of different forces $F_{\gamma}$ and the Stokes drag $F_{\mathrm{SD}}$; $1=$ added mass Faxén correction $\left(F_{\mathrm{AMF}}\right), 2=$ Basset force Faxén correction $\left(F_{\text {BasF }}\right), 3=$ Stokes drag Faxén correction $\left(F_{\mathrm{SDF}}\right), 4=\operatorname{added}$ mass $\left(F_{\mathrm{AM}}\right)$, $5=$ pressure gradient $\left(F_{\mathrm{PG}}\right)$, and $6=$ Basset force $\left(F_{\mathrm{Bas}}\right)$. Particles with $\mathrm{St}=1.38$ and $\rho^{*}=25$ (L5 in Table I) are tracked in stratified turbulence.

of light inertial particles. Both effects can be caused by the different forces that are acting on the particles, and the influence of these forces on light particle dispersion will therefore be investigated in this section.

The computation of all the different forces in the Maxey-Riley equation is an expensive time- and memory consuming job. Therefore, assumptions are often made regarding the forces that can be neglected in the study of particle dispersion. The number of studies underpinning these assumptions, however, is rather limited. An overview of the work on the different terms in the Maxey-Riley equation and their numerical implementation can be found in the paper by Loth. $^{21}$

The term that is most often neglected is the history term because of its numerical complexity. Next to the form as given in Eq. (2), which is derived by Basset, ${ }^{22}$ several other kernels are proposed. Their main difference is the description of the long-time decay rate [other than $t^{-1 / 2}$ as used in Eq. (2)], which is found to depend on the type of flow (see, for example, Mei, ${ }^{23}$ Magnaudet and Eames, ${ }^{24}$ Kim et al. ${ }^{25}$ and Dorgan and Loth $^{26}$ ). We will come back to this point in Sec. III C. Most studies conclude that the importance of the Basset force compared with the other relevant forces is nonnegligible. It can affect the motion of a sedimenting particle, ${ }^{27}$ alter the particle velocity in an isotropic turbulent or oscillating flow field ${ }^{28,29}$ or modify the trapping of particles in vortices. ${ }^{15}$ Nevertheless, the overall effect of the Basset history force on particle dispersion is often found to be small. ${ }^{14,15}$

Before investigating the influence of the forces on the particle dispersion, first the magnitude of the different forces is studied for light particles in stratified turbulence. At every time step the magnitude of the terms in the Maxey-Riley equation, computed as

$$
F_{\gamma} \equiv\left|\boldsymbol{F}_{\gamma}\right|=\left(F_{\gamma, x}^{2}+F_{\gamma, y}^{2}+F_{\gamma, z}^{2}\right)^{1 / 2}
$$

is compared with the Stokes drag. Here, $\gamma$ denotes one of the forces: Stokes drag $F_{\mathrm{SD}}$, pressure gradient, Stokes drag Faxén correction, Basset, Basset Faxén correction, added mass or added mass Faxén correction. A probability density function (PDF) of the ratio of $F_{\gamma} / F_{\mathrm{SD}}$ is shown in Fig. 4 for the particles denoted L5 in Table I. For light particles with $\rho^{*}=\mathcal{O}(1)$ all forces are found to be important, except for the added mass Faxén correction term. With increasing $\rho^{*}$ the relevance of the different forces compared with the Stokes drag diminishes. For the particle parameters shown in Fig. 4 $\left(\rho^{*}=25\right)$ mainly the pressure gradient and the Basset force are important, apart from the Stokes drag.

The question is now whether the relevance of the different forces also emerges for particle dispersion. The forces act at the smallest scales. Because the vertical motions of the particles in stratified turbulence are much smaller than in the horizontal direction, or than in isotropic turbulence, it is expected that the effect of the forces on the particles is more easily noticeable here. A connection between the different forces that act on a light particle and the particle's dispersion behavior is made by performing a simulation in which some of the forces are switched off. In a single run five types of particles are tracked, with 4000 particles of each type. The first are fluid particles (denoted "fluid" in Fig. 5) and the second are the particles denoted L5 in Table I (and in Fig. 5). The other particles also have the properties of particles L5, but now not all the forces in the Maxey-Riley equation are incorporated. For the third group of particles the two Basset force terms are set equal to zero (P). For the fourth group all Faxén correction terms are set to zero (Q) and for the last
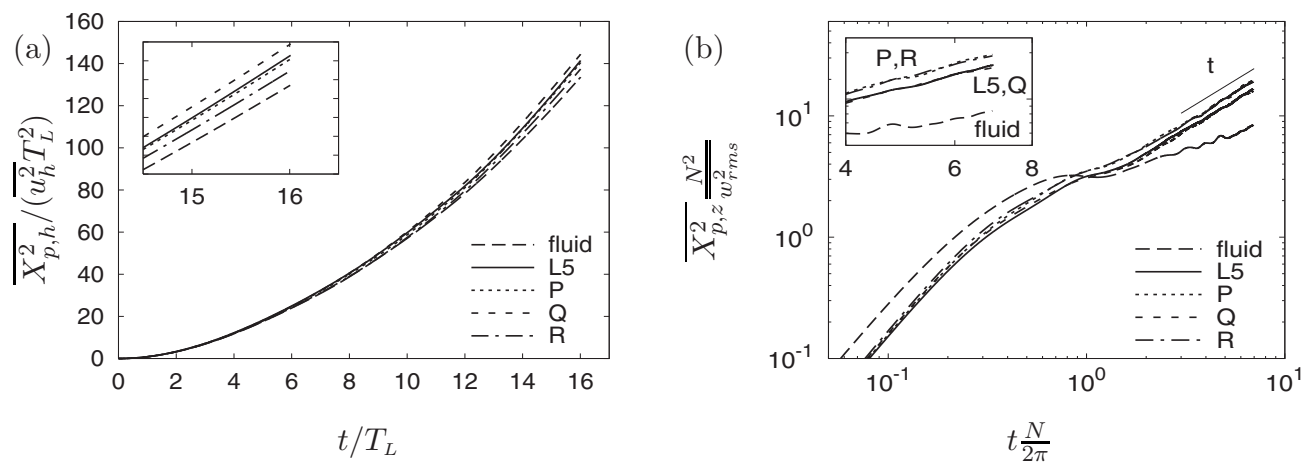

FIG. 5. Mean-squared displacement of fluid particles and light particles in (a) horizontal and (b) vertical direction in stratified turbulence. For the particles denoted with L5 all the forces are incorporated except for gravity. For particles P the Basset forces are switched off for particles Q all Faxén correction terms are set equal to zero and for particles R only the Stokes drag, the pressure gradient and the added mass force are taken into account (see Table II for an overview). No gravity is acting on the particles. 
TABLE II. Properties of the particles that are used to study the influence of the different forces on particle dispersion. For all particle types $\rho^{*}=25.0$ and $\mathrm{St}=1.38$. The forces are $F_{\mathrm{SD}}=$ Stokes drag, $F_{\mathrm{SDF}}=$ Stokes drag Faxén correction, $F_{\mathrm{PG}}=$ pressure gradient, $F_{\mathrm{AM}}=$ added mass, $F_{\mathrm{AMF}}=$ added mass Faxén correction, $F_{\text {Bas }}=$ Basset, $F_{\text {BasF }}=$ Basset Faxén correction, and $F_{g}=$ gravity.

\begin{tabular}{lcccccccc}
\hline \hline Particle & $F_{\mathrm{SD}}$ & $F_{\mathrm{SDF}}$ & $F_{\mathrm{PG}}$ & $F_{\mathrm{AM}}$ & $F_{\mathrm{AMF}}$ & $F_{\text {Bas }}$ & $F_{\text {BasF }}$ & $F_{g}$ \\
\hline $\mathrm{L} 5$ & $\mathrm{x}$ & $\mathrm{x}$ & $\mathrm{x}$ & $\mathrm{x}$ & $\mathrm{x}$ & $\mathrm{x}$ & $\mathrm{x}$ & \\
$\mathrm{P}$ & $\mathrm{x}$ & $\mathrm{x}$ & $\mathrm{x}$ & $\mathrm{x}$ & $\mathrm{x}$ & & & \\
$\mathrm{Q}$ & $\mathrm{x}$ & & $\mathrm{x}$ & $\mathrm{x}$ & & $\mathrm{x}$ & & \\
$\mathrm{R}$ & $\mathrm{x}$ & & $\mathrm{x}$ & $\mathrm{x}$ & & & & \\
$\mathrm{W}=0.05$ & $\mathrm{x}$ & $\mathrm{x}$ & $\mathrm{x}$ & $\mathrm{x}$ & $\mathrm{x}$ & $\mathrm{x}$ & $\mathrm{x}$ & $\mathrm{x}$ \\
$\mathrm{S}$ & $\mathrm{x}$ & $\mathrm{x}$ & $\mathrm{x}$ & $\mathrm{x}$ & $\mathrm{x}$ & & & $\mathrm{x}$ \\
\hline \hline
\end{tabular}

group these two effects are combined (R). The forces acting on the fifth group of particles are thus the Stokes drag, the pressure gradient and the added mass. Which forces act on the various particles is summarized in Table II. These choices are based on the approximations that are commonly made in the literature. It is chosen to study particles with a density ratio of 25.0, because their dispersion behavior shows deviations from that of fluid particles, but at the same time also other forces than the drag force are relevant. The dispersion of these five types of particles is shown in Fig. 5. The results from the light particles indeed differ from those obtained for the fluid particles, which is most clear in Fig. 5(b) (cf. Fig. 2). In the horizontal direction small differences (less than 3\%) between the four groups of inertial particles can be seen in the long-time limit. In the vertical direction, on the other hand, clear differences can be observed. In the inset of Fig. 5(b) the long-time behavior is highlighted. The results for the particles for which all the Faxén correction terms are switched off are the same as those for the benchmark particles L5. For the other two groups of particles the vertical dispersion is increased compared with that of the particles L5. This increase is about 15\%-20\%. It can thus be concluded that the Basset force does play a role in the vertical dispersion of light inertial particles in stably stratified turbulence. This influence has two causes. The vertical motion of the particles in stratified turbulence occurs on much smaller scales, and, moreover, the oscillatory wavelike motion induces considerable accelerations and decelerations.

The Basset force has a restoring effect on the particles; it tends to reduce the velocity difference between a particle and the fluid. ${ }^{15,16,19}$ Indeed, neglecting this force results in an overprediction of the vertical particle dispersion.

\section{Discussion}

In order to investigate whether the influences of the Basset force on inertial particle dispersion are unique for stratified turbulence and for light particles, the same procedure as followed in Sec. III B is applied for isotropic turbulence and for heavy particles. Furthermore, the influence of the lift force or gravity on the particles will be examined and it is discussed whether the assumptions underlying the Maxey-Riley equation are met for the light particles used in this work. We will conclude with a discussion on the model chosen for the history force.

\section{Isotropic turbulence}

In isotropic turbulence $\left(\operatorname{Re}_{\lambda}=85\right)$ the dispersion statistics are computed for light particles with $d_{p}=2 \eta, \rho^{*}=4.0$, and $\mathrm{St}=0.86$. The difference between the particle dispersion with inclusion of all the forces and with only a subset of the forces is only about $2 \%$. Moreover, whether switching off a certain force has an increasing or decreasing effect on the dispersion differs per spatial component. It can thus be concluded that the assumptions made in the literature, where the Basset force and the Faxén correction terms are often neglected in dispersion studies, are justified for isotropic turbulence and particle parameters comparable to those investigated here. The relative importance of the different forces (cf. Fig. 4), however, is very comparable in isotropic turbulence and in stratified turbulence. This strong resemblance is due to the fact that the forces act at the smallest scales of the flow, which are also more or less isotropic for moderately stratified turbulence.

\section{Heavy particles}

For heavy particles the drag force is by far the largest force that acts on a particle. When the full Maxey-Riley equation is solved for heavy particles instead of the simplified version Eq. (3), it is found that the Basset force is a factor about 10 smaller than $F_{\mathrm{SD}}$, compared with a factor of about 2 for light particles (cf. Fig. 4). Heavy particles with the same Stokes number as the light particles discussed in Sec. III B $(\mathrm{St}=1.38)$, and with $d_{p}=0.04 \eta, \rho^{*}=13500$ are used to study the influence of the Basset force on heavy particle dispersion in stratified turbulence. Now, the difference between the results obtained with and without inclusion of the Basset force is negligible.

\section{Particle size and particle Reynolds number}

A remark has to be made regarding the choice of the particle size. For the Maxey-Riley equation to be valid, $d_{p}$ must be smaller than the Kolmogorov scale $\eta$. Here, we have chosen $d_{p} \lesssim \eta$ in order to obtain Stokes numbers of up to $\mathcal{O}(1)$. Another requirement for the Maxey-Riley equation is that the particle Reynolds number $\operatorname{Re}_{p}$ is smaller than one. The light particles considered in this paper not all satisfy this requirement. The mean value of $\mathrm{Re}_{p}$ for the heaviest particles (L5) is about 1.5. A future study on the choice of the drag law (linear or nonlinear) can be interesting, although the effect of the type of drag law on the conclusions drawn in this paper is expected to be small because of the relatively low Reynolds numbers.

\section{Lift force}

In stratified turbulence large vertical shear is present locally. Therefore, in a separate study, the effect of the Saffman lift force is examined. For heavy particles the influence of the lift force on the vertical dispersion can be neglected, as described in a previous paper. ${ }^{8}$ For light particles the magnitude of the lift force compared with the Stokes drag force or the Basset force is found to be an order of magnitude 


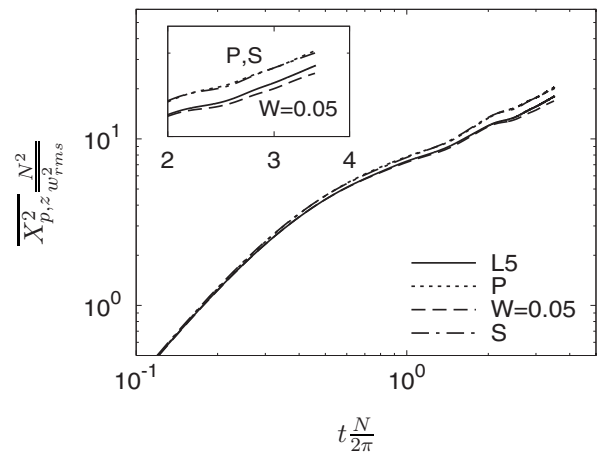

FIG. 6. Vertical single-particle dispersion for light particles with $\mathrm{St}=1.38$ and $\rho^{*}=25$, under the influence of gravity on the particles. The mean displacement resulting from the settling velocity is subtracted. See Table II for an overview of which forces are acting on the different particles. Both without gravity (pair L5, P) and with gravity (pair W=0.05, S) the vertical dispersion is larger when the Basset forces are switched off.

smaller. Also its influence on vertical dispersion is found to be negligible. ${ }^{19}$ For this reason no lift forces are included in the present study.

\section{Gravity}

In all practical situations of course gravity acts on the individual particles. As a result the particles will obtain a mean settling velocity in the vertical direction. This settling velocity $w_{\text {st }}$ will be related to the vertical rms-velocity of the fluid, by using $\mathrm{W}=w_{\mathrm{st}} / w_{\text {rms }}$. The influence of the Basset force is studied for particles with $W=0.05$. According to the results for heavy particles (see Ref. 8), the vertical dispersion of these particles is expected to deviate from the dispersion without gravity. A value of $\mathrm{W}=0.05$ would be reasonable for marine particles with density ratios of $\mathcal{O}(1)$ and diameters of about $10^{-4}<d_{p}<10^{-5} \mathrm{~m}$.

For the simulations including gravity 20000 particles are tracked, on the expense of a shorter time range. The results are shown in Fig. 6. Particles denoted L5 and P display the same result as in Fig. 5; without the Basset forces the vertical dispersion is about $15 \%$ larger for long times. This confirms that the 4000 light particles used for the results in the previous sections are enough to compute statistics. For the particles on which gravity acts as a body force (denoted $\mathrm{W}=0.05$ and $\mathrm{S}$ in Fig. 6) the dispersion after subtraction of the mean vertical displacement resulting from the settling velocity is shown (see Ref. 8 for clarification). Again the vertical dispersion without incorporation of the Basset force is larger, by about $18 \%$. The conclusion that the Basset force needs to be taken into account when studying the vertical dispersion of light inertial particles remains valid when gravity acts on the particles.

\section{History force kernel}

As mentioned in Sec. III B, other kernels are proposed for the history force. Mei and Adrian ${ }^{30}$ proposed a $(t-\tau)^{-2}$ decay at large times, which corresponds fairly well with several test cases. ${ }^{23}$ The original kernel, with $(t-\tau)^{-1 / 2}$ for both short and long times, might therefore overpredict the magnitude of the history force. In order to investigate the depen- dence of our results on the kernel in the history force, the simulation presented in Sec. III B is repeated using Eq. (9) in Ref. 23 for the history force $\left[(t-\tau)^{-2}\right.$ decay for large times]. Unfortunately, solving the equation of motion for the particles now becomes even more time consuming, and could only be solved for a short time range. The results, however, show the same trend as the results obtained with Basset's description of the history force. The magnitude of the force is similar-although time series of the history force show smaller peaks. Also the observation that the vertical dispersion with inclusion of the history force is smaller than without is reproduced. The difference between lines L5 and P, Q as presented in Fig. 5 is a little smaller now, but still significant.

For oscillatory motion with low frequency the integration kernel is found to be better described by exponential decay for large time. ${ }^{31}$ The flow parameters of our stably stratified turbulence (in which wavelike motion is present), however, result in Strouhal numbers $\mathrm{Sr}_{\omega} \geq \mathrm{Re}$, indicating that this exponential decay law is not applicable here. ${ }^{31}$ Furthermore, it is stated in literature (see, for example, Refs. 23 and 24) that for a particle that reverses impulsively-resembling the vertical oscillating behavior of particles in stratified turbulence-a better description is a long-time decay scaling as $(t-\tau)^{-1}$, which is closer to the original $(t-\tau)^{-1 / 2}$ behavior.

The conclusion that the history force needs to be included when studying light particle dispersion in stratified turbulence is thus independent of the choice of the history force kernel.

\section{CONCLUDING REMARKS}

The numerical study of light particle dispersion in forced stably stratified turbulence is reported. To this end the full Maxey-Riley equation is solved as the equation of motion for the particles. The influence of the background density gradient is taken care of by making the density ratio $\rho^{*}$ timedependent and by keeping the particles away from the domain boundaries.

The dispersion behavior is mainly determined by the Stokes number of the particle, as shown by the comparison with heavy particle dispersion in stratified turbulence. Just as for heavy particle dispersion, horizontal light particle dispersion in stratified turbulence displays long-time scaling behavior proportional to $\mathcal{O}\left(t^{2}\right)$. In the vertical direction the plateau is shorter for the light inertial particles than for fluid particles, even for small Stokes numbers. For larger Stokes numbers it is again found that increasing St results in a less pronounced plateau. In the long-time limit, the vertical dispersion increases with increasing St and the scaling behavior becomes proportional to $t$. Whereas this diffusive regime is caused by molecular diffusion of the active scalar (density) for fluid particles for inertial particles with $\mathrm{St} \geq \mathcal{O}(1)$ it is a statistical, random walklike property; the particles become uncorrelated from their initial position.

Several forces act on the light particles, of which the drag force, the pressure gradient and the Basset force are the most important. The (Basset) history force is often neglected in literature because of its numerical complexity. We have 
shown that for light particle dispersion in stably stratified turbulence, with modified Reynolds number $R=\mathrm{Fr}_{h}^{2} \mathrm{Re}=4.7$, this history force needs to be taken into account. Neglecting this force results in an overprediction of the vertical dispersion of about $15 \%-20 \%$. This result is obtained both with and without taking into account the gravity force on the particles. The influence of the history force on vertical dispersion is also obtained for other choices of the history force kernel than the original one proposed by Basset. Furthermore, we presented that this effect is unique for light particles in stratified turbulence; for light particle dispersion in isotropic turbulence or for heavy particle dispersion the history force can indeed be neglected for the parameter range studied here. A few reasons can be identified why the influence of the Basset force is so strong for particles in stratified turbulence. For small $\rho^{*}$ the history force has a comparable magnitude as the drag force, and the vertical motions in stratified turbulence are much smaller than in isotropic turbulence. Moreover, the particles undergo large accelerations and decelerations during their oscillating motion.

\section{ACKNOWLEDGMENTS}

This program is funded by the Netherlands Organisation for Scientific Research (NWO) and Technology Foundation (STW) under the Innovational Research Incentives Scheme Grant No. ESF.6239. This work is sponsored by the Stichting Nationale Computerfaciliteiten (NCF, NWO) for the use of supercomputer facilities. The authors would like to thank Professor Jacques Magnaudet (IMFT Toulouse, France) for valuable suggestions.

${ }^{1}$ J. E. Cloern, "Phytoplankton bloom dynamics in coastal ecosystems: A review with some general lessons from sustained investigation of San Francisco Bay, California," Rev. Geophys. 34, 127, doi:10.1029/ 96RG00986 (1996).

${ }^{2}$ J. J. Riley and M.-P. Lelong, "Fluid motions in the presence of strong stable stratification," Annu. Rev. Fluid Mech. 32, 613 (2000).

${ }^{3}$ G. Brethouwer, P. Billant, E. Lindborg, and J.-M. Chomaz, "Scaling analysis and simulation of strongly stratified turbulent flows," J. Fluid Mech. $\mathbf{5 8 5}, 343$ (2007).

${ }^{4}$ M. van Aartrijk, H. J. H. Clercx, and K. B. Winters, "Single-particle, particle-pair and multi-particle dispersion of fluid particles in forced stably stratified turbulence," Phys. Fluids 20, 025104 (2008).

${ }^{5}$ L. Liechtenstein, F. S. Godeferd, and C. Cambon, "The role of nonlinearity in turbulent diffusion models for stably stratified and rotating turbulence," Int. J. Heat Fluid Flow 27, 644 (2006).

${ }^{6}$ Y. Kimura and J. R. Herring, "Diffusion in stably stratified turbulence," J. Fluid Mech. 328, 253 (1996).

${ }^{7}$ F. Nicolleau and J. C. Vassilicos, "Turbulent diffusion in stably stratified nondecaying turbulence," J. Fluid Mech. 410, 123 (2000).

${ }^{8}$ M. van Aartrijk and H. J. H. Clercx, "Dispersion of heavy particles in stably stratified turbulence," Phys. Fluids 21, 033304 (2009).
${ }^{9}$ G. I. Taylor, "Diffusion by continuous movements," Proc. London Math. Soc. s2-20, 196 (1922).

${ }^{10}$ H. J. Pearson, J. S. Puttock, and J. C. R. Hunt, "A statistical model of fluid-element motions and vertical diffusion in a homogeneous stratified turbulent flow," J. Fluid Mech. 129, 219 (1983).

${ }^{11}$ E. Lindborg and G. Brethouwer, "Vertical dispersion by stratified turbulence," J. Fluid Mech. 614, 303 (2008).

${ }^{12}$ M. R. Maxey and J. J. Riley, "Equations of motion for a small rigid sphere in a nonuniform flow," Phys. Fluids 26, 883 (1983).

${ }^{13}$ M. van Aartrijk and H. J. H. Clercx, "Preferential concentration of heavy particles in stably stratified turbulence," Phys. Rev. Lett. 100, 254501 (2008).

${ }^{14} \mathrm{~V}$. Armenio and V. Fiorotto, "The importance of the forces acting on particles in turbulent flows," Phys. Fluids 13, 2437 (2001).

${ }^{15} \mathrm{P}$. Tanga and A. Provenzale, "Dynamics of advected tracers with varying buoyancy," Physica D 76, 202 (1994).

${ }^{16}$ F. Candelier, J. R. Angilella, and M. Souhar, "On the effect of the Boussinesq-Basset force on the radial migration of a Stokes particle in a vortex," Phys. Fluids 16, 1765 (2004).

${ }^{17}$ K. B. Winters, J. A. MacKinnon, and B. Mills, "A spectral model for process studies of rotating, density-stratified flows," J. Atmos. Ocean. Technol. 21, 69 (2004).

${ }^{18}$ P. Gentien, P. L. Donaghay, H. Yamazaki, R. Raine, B. Reguera, and T. R. Osborn, "Harmful algal blooms in stratified environments," Oceanogr. 18, 172 (2005).

${ }^{19}$ M. van Aartrijk, "Dispersion of inertial particles in stratified turbulence," Ph.D. thesis, Eindhoven University of Technology, 2008.

${ }^{20}$ T. R. Auton, J. C. R. Hunt, and M. Prud'homme, "The force exerted on a body in inviscid unsteady nonuniform rotational flow," J. Fluid Mech. 197, 241 (1988).

${ }^{21}$ E. Loth, "Numerical approaches for motion of dispersed particles, droplets and bubbles," Prog. Energy Combust. Sci. 26, 161 (2000).

${ }^{22}$ A. B. Basset, A Treatise on Hydrodynamics (Deighton, Bell and Co., Cambridge, 1888), Vol. 2.

${ }^{23}$ R. Mei, "Velocity fidelity of flow tracer particles," Exp. Fluids 22, 1 (1996).

${ }^{24}$ J. Magnaudet and I. Eames, "The motion of high-reynolds-number bubbles in inhomogeneous flows," Annu. Rev. Fluid Mech. 32, 659 (2000).

${ }^{25}$ I. Kim, S. Elghobashi, and W. A. Sirignano, "On the equation for spherical-particle motion: effect of Reynolds and acceleration numbers," J. Fluid Mech. 367, 221 (1998).

${ }^{26}$ A. J. Dorgan and E. Loth, "Efficient calculation of the history force at finite Reynolds numbers," Int. J. Multiphase Flow 33, 833 (2007).

${ }^{27}$ Y. D. Sobral, T. F. Oliveira, and F. R. Cunha, "On the unsteady forces during the motion of a sedimenting particle," Powder Technol. 178, 129 (2007).

${ }^{28}$ R. Mei, R. J. Adrian, and T. J. Hanratty, "Particle dispersion in isotropic turbulence under Stokes drag and Basset force with gravitational settling," J. Fluid Mech. 225, 481 (1991).

${ }^{29}$ D. J. Vojir and E. E. Michaelides, "Effect of the history term on the motion of rigid spheres in a viscous fluid," Int. J. Multiphase Flow 20, 547 (1994).

${ }^{30}$ R. Mei and R. J. Adrian, "Flow past a sphere with an oscillation in the free-stream velocity and unsteady drag at finite Reynolds number," J. Fluid Mech. 237, 323 (1992).

${ }^{31}$ P. M. Lovalenti and J. F. Brady, "The force on a sphere in a uniform flow with small-amplitude oscillations at finite Reynolds number," J. Fluid Mech. 256, 607 (1993). 